\title{
The interplay of lipids, lipoproteins and immunity in atherosclerosis
}

\section{Angela Pirillo $^{1,2}$, Fabrizia Bonacina ${ }^{3}$, Giuseppe Danilo Norata ${ }^{3,4}$, Alberico Luigi Catapano ${ }^{2,3}$}

${ }^{1}$ Center for the Study of Atherosclerosis, Bassini Hospital, Cinisello Balsamo, Italy

${ }^{2}$ IRCCS Multimedica, Milan, Italy

${ }^{3}$ Department of Pharmacological and Biomolecular Sciences, Università degli Studi di Milano, Milan, Italy

${ }^{4}$ School of Biomedical Sciences, Curtin Health Innovation Research Institute, Curtin University, Perth, Western Australia.

\section{Corresponding Author}

\section{Alberico L. Catapano}

Department of Pharmacological and Biomolecular Sciences, University of Milan and IRCCS Multimedica Via Balzaretti, 9 - 20133 Milan ITALY

Phone number: +39250318302

Fax number: +39250318386

e-mail: alberico.catapano@unimi.it 


\section{Abstract}

Purpose of Review. Atherosclerosis is an inflammatory disorder of the arterial wall, in which several players contribute to the onset and progression of the disease. Beside the well established role of lipids, specifically cholesterol, and immune cell activation, new insights on the molecular mechanisms underlying the atherogenic process have emerged.

Recent Findings. Meta-inflammation, a condition of low-grade immune response caused by metabolic dysregulation, alteration of the non-classical immunological memory of innate immune cells (referred to as "trained immunity"), cholesterol homeostasis in dendritic cells and immunometabolism, i.e. the interplay between immunological and metabolic processes, have all emerged as new actors during atherogenesis. These observations reinforced the interest in directly targeting inflammation to reduce cardiovascular disease.

Summary. The novel acquisitions in pathophysiology of atherosclerosis reinforce the tight link between lipids, inflammation and immune response, and support the benefit of targeting LDL-C as well as inflammation to decrease the CVD burden. How this will translate into the clinic will depend on the balance between costs (monoclonal antibodies either to PCSK9 or to IL-1ß), side effects (increased incidence of death due to infections for anti-IL-1ß antibody) and the benefits for patients at high risk.

Keywords: Atherosclerosis, cholesterol, inflammation, immune response 


\section{Inflammation, immune response and atherosclerosis: which role for lipids and lipoproteins?}

Atherosclerosis is a pathological condition characterized by a chronic, non-resolving low-grade inflammation within the arterial wall resulting from the accumulation of apoB-containing lipoproteins in sites where disturbed laminar flow is present [1]. This event triggers the recruitment of monocytemacrophages, the most abundant immune cells present in atherosclerotic lesions, and cells of adaptive immune response such as T lymphocytes [1].

Aim of this review is to discuss the emerging concepts in the field of immunity and atherosclerosis with a main focus on the interplay among lipids, cellular metabolic functions and immune cells activation.

Cholesterol is an essential component of cellular membranes; lipid rafts are microdomains of the plasma membrane that are enriched in cholesterol and sphingolipids and serve as a platform in which proteins involved in signal transduction pathways transiently converge to trigger a cellular response [2]. In the context of atherosclerosis, lipid rafts are involved in the regulation of several processes including immune cell activation, inflammation, and proliferation of smooth muscle cells [2]. Alterations in the lipid composition of lipid rafts, and in particular in their cholesterol content, following the interaction with plasma lipoproteins, may lead to changes of lipid raft-dependent signaling; such alterations include either cholesterol enrichment, triggered by the cholesterol transported in LDL, or cholesterol depletion, induced by HDL [2]. LDL and oxidized LDL (specifically oxysterols) increase the formation of lipid rafts, which in turn support the translocation of proteins including NADPH oxidase, transient receptor potential channel-1 (TRPC-1) or endothelial nitric oxide synthase, all leading to increased oxidative stress, calcium-mediated apoptosis, secretion of pro-inflammatory cytokines and inhibition of nitric oxide secretion [2]; conversely, HDL-mediated-raft reduction contributes to the maintenance of endothelial integrity [2-5]. Lipid rafts also play a role in immune cells signaling by assisting the formation of immunological synapses, specialized membrane regions in which receptors essential for immune cell activity, including toll like receptors (TLRs), T cell receptor (TCR) and $B$ cell receptor $(B C R)$ as well as major histocompatibility complex $(\mathrm{MHC}$ ) molecules are located [6]. Studies on lymphocytes showed that aggregation of membrane rafts facilitates cell signaling after TCR ligation [7] and that accumulation of cholesterol in T cells from patients with systemic lupus erythematosus lowered the threshold for activation and TCR signaling leading to aberrant immune response [8]. Lipid rafts are also involved in antigen presentation by harboring $\mathrm{MHCll}$ and thus playing a key role in APC-mediated T cell activation and proliferation [9]. Disruption of these domains alters MHCII localization in raft microdomains and dramatically inhibits antigen presentation [10]; similar findings were reported in macrophages where selective deficiency of ATB-binding cassette transporter A1 (ABCA1) leads to increased MyD88-dependent TLRs recruitment to lipid rafts [11] thus changing macrophage toward an hyper-responsive phenotype to LPS challenge.

The imbalance between the rate of cholesterol uptake and the rate of cholesterol removal through efflux mechanisms in immune cells, however, does not only affect lipid rafts biology but also favors the 
accumulation of cholesteryl esters (CE) [12]. This may occur already in circulating mononuclear cells which become enriched in cholesterol when plasma levels of LDL are high and levels of HDL are low. Cholesterolenriched monocytes are more likely to adhere to a damaged endothelium and migrate into the intima [1315], where they differentiate into macrophages. Indeed, lipid accumulation in monocytes, which has been observed in the postprandial phase and associated with increased activation in individuals with metabolic syndrome, may promote atherogenesis by facilitating their adhesion and transendothelial migration [16]. Here, macrophages may further take up modified lipoproteins through scavenger receptors and become foam cells. Following the uptake of lipoproteins, CE present in the core are hydrolyzed to free cholesterol $(\mathrm{FC})$; in the presence of excess $\mathrm{FC}$, which can induce membrane damage and cytotoxicity, compensatory mechanisms are activated to reduce cholesterol synthesis and uptake and increase cholesterol efflux [17]. When these mechanisms fail, FC is re-esterified for storage in lipid droplets, which are metabolically active intracellular organelles composed by a hydrophobic core of neutral lipids surrounded by a phospholipid monolayer coated with specific proteins and are believed to contribute to the development of atherosclerosis $[17,18]$.

Although this mechanism is initially protective, the continuous accumulation of lipid droplets may shift macrophages to a long term pro-inflammatory phenotype. This profile appears to be the consequence of the acquisition by innate immune cells of an memory which differs from what observed in cells of the adaptive arm of the immune system $[19,20]$. This phenomenon is referred to as "trained immunity" or innate immune memory (Figure 1). Trained immunity is based on epigenetic reprogramming, i.e. sustained changes in transcription programs following changes in chromatin organization, histone modifications and persistence of microRNAs induced by the first exposure to the dangerous stimulus [19]. This leads to an increased pro-inflammatory response and may help to explain the basis of the well established relationship between infection and atherosclerosis [21]. In the context of atherosclerosis, trained immunity may be induced also by non-microbial stimuli, such as oxidized LDL (OxLDL) or lipoprotein(a) $[\operatorname{Lp}(a)][22,23]$. Experimental data suggest that a brief stimulation of monocytes with low concentrations of OxLDL (but not LDL) results in epigenetic histone modifications leading to increased production of pro-inflammatory cytokines and foam cell formation following re-stimulation [22]. The activation of trained immunity appears to be also related to $L p(a)$. Indeed subjects with elevated $L p(a)$ levels not only exhibit increased arterial inflammation, but also present monocytes which remain in a long-lasting primed state characterized by an enhanced capacity to adhere and transmigrate the endothelium and increased production of proinflammatory cytokine following TLR stimulation [23]. In addition, Lp(a) primes monocytes toward a responsive state, resulting in an increased production of pro-inflammatory cytokines upon restimulation [23]. Due to the short half-life of monocyte-macrophages, the concept of trained immunity implies that such epigenetic changes must be maintained by similar changes in hematopoietic precursor cells in the bone marrow [24]. Altogether these observations suggest that, although trained immunity may represent a 
valuable way to protect the host against recurrent infections, it might contribute and even accelerate the development of atherosclerosis. Cholesterol crystals accumulation in the extracellular space may damage and activate intimal surface, thus resulting in the production of pro-inflammatory cytokines that promote the growth as well as atherosclerotic plaque destabilization [25-27].

\section{Emerging immuno-players in atherosclerosis}

At the molecular level, the enrichment of lipid rafts with cholesterol leads to the formation of cholesterol crystalline membrane domains which precedes the release of cholesterol crystal in the extracellular space [28]. Cholesterol crystals initiate inflammation via NLRP3 inflammasome activation, leading to the secretion of IL-1ß [29]. The inflammasome is a cytoplasmic complex containing multiple proteins, which is formed in response to damage/danger-associated molecular patterns (DAMPs) or pathogen-associated molecular patterns (PAMPs) expressed by macrophages, and serves as a molecular platform for the activation of the cysteine protease caspase-1 [29]. Several types of inflammasome complexes have been reported but only the activation of NLRP3 inflammasome and consequent inflammatory responses contributes to the progression of atherosclerosis [30]. Current data indicate that OxLDL triggers an inflammatory "priming" [31], which is followed by cholesterol crystal-mediated activation of the inflammasome [32, 33]. Causality of this mechanism in atherogenesis has been demonstrated in LDLR KO mice in which the deficiency of NRLP3 in bone marrow cells protects from the disease [34]. Release of IL-1 $\beta$ and IL-18 pro-inflammatory cytokines following NRLP3 activation promotes atherosclerosis and the use of animal models have clearly demonstrated, with few exceptions $[32,35]$, that their deficiency as well as deficiency of caspase-1 reduces the size of atherosclerotic lesions [36-39].

More recently, metabolically-driven inflammation, known as meta-inflammation, was postulated to be critical in explaining the deep connection among obesity, diabetes and atherosclerosis (Figure 2). [40]. Meta-inflammation is a chronic low-grade inflammation connecting alterations in systemic metabolism with the impairment in immune cells cellular metabolism and function [41]. The close relationship between metabolism and immuno-inflammation is further supported by the observation that NLRP3-deficient mice on a high-fat diet become insulin hypersensitive [42], thus suggesting that inflammasome is somehow linked to insulin action; in humans increased levels of LDL-C induce pro-IL-1 $\beta$ production through TLRs [43], and saturated fatty acids, such as palmitate and stearate, activate NLRP3 in primed macrophages in a ROSand AMPK-dependent manner [44]. In mice, dyslipidemia induced by deficiency of key genes involved in cholesterol homeostasis, such as apolipoprotein E or ATP-binding cassette A1 or G1, promotes myelopoiesis and extramedullary hematopoiesis, resulting in increased levels of circulating monocytes and inflammatory response, as the consequence of cholesterol enrichment in lipid rafts of immune cells [4547]. These observations have driven the research toward the investigation of the impact of intracellular lipid alteration in immune cell function, a field known as immunometabolism [41] which shares molecular 
mechanisms of pathogenicity with cardiometabolic diseases, autoimmunity and cancer. Lipid homeostasis is finely regulated by transcription factors namely liver $X$ receptors (LXR) and sterol regulatory-element binding proteins (SREBP) which sense cholesterol and lipid concentration thus promoting lipid removal or biosynthetic pathways respectively [48]. This dichotomy has been widely investigated in macrophages where, following inflammation, de-novo lipogenesis is required to support inflammasome activation [49], and later (12-24 hours following TLR4 activation) plays a key role in the resolution phase by producing antiinflammatory fatty acids [50]. Also the activation of LXR is known to be anti-inflammatory as it represses TLRs 2,4 and 9-dependent NF-KB activation via modulation of ABCA1-mediated cholesterol efflux, lipid raft enrichment and TLRs localization in the membranes [51]. The notion that cholesterol is an important component of cell membrane and is essential for cell growth and proliferation [52] can likewise be applied to the adaptive immune responses where lymphocytes undergo rapid expansion in response to antigenic challenge. Activation of LXR during T cell priming inhibits mitogen-driven expansion, with deficiency of LXR $\beta$ conferring a proliferative advantage [53]; on the contrary, SREBP signaling is critical for membrane synthesis during blastogenesis and T cells expansion [54]. Once proliferated, immune cells migrate from the lymph nodes toward the inflamed tissues where they have to adapt to a different microenvironment with a specific extracellular matrix, growth factors, oxygen, nutrients, and metabolites [55]. As an example, in metabolically demanding environment such as tumors but also in atherosclerotic plaques, infiltrating immune cells change their immunometabolic settings and function [55]. Indeed, tissue/local enrichment of certain metabolites affects immune cell function as in the case of i) lactic acid, which is produced during hypoxia at the site of inflammation and contribute to disrupting $T$ cell motility and reducing cytolytic function of CD8 T cells [56], or of ii) saturated fatty acid which are released by adipose tissue and differently affect differentiation and trafficking patterns of CD4 T cells [57].

Dendritic (DCs) cells play a key role in antigen processing and presentation and are therefore fundamental in connecting innate and adaptive immune response [58]. DCs migrate to lymphoid tissues to support the priming of selective antigen-reactive T cell clones [59] and actively participate in all stages of atherogenesis from fatty streaks to mature lesions formation [60]. DCs undergo an extensive differentiation program where balance of intracellular metabolism plays a crucial role. Lipid overload in DCs which are deficient in ABCA1/ABCG1 exacerbates autoimmune disease [12] and inflammasome activation [61], thus suggesting that cholesterol efflux pathways in these cells play a key role in the maintenance of immune tolerance. These findings suggest that the removal of cholesterol excess could be considered a therapeutic target to limit the immuno-inflammatory response in the context of atherosclerosis and also of autoimmune diseases where HDL are less efficient in promoting cholesterol efflux [62, 63]. In vitro, stimulation of HDLdependent reverse cholesterol transport disrupt lipid rafts, attenuate antigen presentation in DCs [64] and ameliorate autoimmune disease in mice [12]. Whether targeting cholesterol mobilization in immune cells 
will transfer in beneficial effect in the context of atherosclerosis, beyond the null effect of increasing plasma HDL-C levels, remains to be addressed.

\section{Targeting inflammation to reduce cardiovascular burden: a lesson from the clinical trials}

The key role of inflammation in atherosclerosis supports the hypothesis that targeting the immuneinflammatory response will have a beneficial impact on disease outcome.

Is there scientific evidence supporting this hypothesis?

Statins, by promoting LDL-R expression and LDL-C reduction, demonstrated a significant and robust benefit in terms of cardiovascular risk reduction $[65,66]$ and effect also ascribed to their ability to decrease circulating inflammatory markers such as CRP and to improve endothelial function and plaque burden, effects which were associated to their potential pleiotropic activities [67]. Despite a large interindividual variability in LDL-C reductions following statin therapy [68], an extensive meta-analysis of clinical trials with statins showed the tight dependence of CRP reduction on LDL-C reduction [69]. Studies with other lipid lowering drugs such as ezetimibe showed that the beneficial effect of LDL-C-lowering therapies on systemic inflammatory status, as monitored by changes in CRP plasma levels, could be achieved, independently of the mechanism of action, mainly in patients already presenting with baseline inflamed conditions [70]. More importantly, Mendelian randomization studies have demonstrated that CRP is not a causal factor for atherosclerosis but a rather aspecific marker of systemic inflammation [71-75]. In many clinical trials with PCSK9 inhibitors, including the large outcome trial with evolocumab (FOURIER) [76], baseline levels of CRP were below $2 \mathrm{mg} / \mathrm{L}$ [70]. In the FOURIER study, neither the treated arm nor the placebo arm experienced a reduction of CRP compared to baseline in spite of a substantial LDL-C reduction in the treated arm [76]. A similar trend was observed in many other trials where, in patients with CRP below $2 \mathrm{mg} / \mathrm{L}$ at baseline, LDLC-lowering therapies, from statins to ezetimibe to anti-PCSK9 antibodies, did not affect CRP levels, perhaps because these patients did not present a relevant systemic inflammation [70]. Of note, even in patients with a CRP is below $2 \mathrm{mg} / \mathrm{L}$ and not altered by the therapy, such as in the GLAGOV study, LDL-C reduction with anti-PCSK9 antibodies results in atherosclerotic plaque regression as determined by IVUS [77]. These observations strengthen the direct link between cholesterol and atherosclerosis and indicate that decreasing LDL levels is one of the key goals for improving cardiovascular outcome beyond tuning inflammation.

Do we have evidence for anti-inflammatory treatments on cardiovascular outcome? While most of the studies with anti-inflammatory agents failed to show a relevant benefit in terms of cardiovascular risk reduction, the results from the CANTOS trial with a monoclonal antibody (canakinumab) targeting IL-1ß demonstrated a benefit on cardiovascular outcome [78]. Patients with a previous myocardial infarction and 
an elevated level of high-sensitivity C-reactive protein received one of three doses of canakinumab (50, 150 and $300 \mathrm{mg}$ ) or placebo [78]. The treatment with the antibody $(150 \mathrm{mg})$ resulted in a significant lower incidence of the primary end point (a composite of nonfatal myocardial infarction, nonfatal stroke, and cardiovascular death) vs placebo [78]. The reduction was mainly driven by a reduction in the incidence of myocardial infarction [78]. The benefit occurred in the absence of a difference in the LDL-C among groups. How can we reconcile these observations? Targeting IL-1ß is downstream of the inflammasome activation driven by cholesterol. Therefore, a cholesterol driven pathway is addressed with this therapy therefore not surprisingly the results are similar to what can be expected by a cholesterol reduction in these patients. Indeed, would have been very informative if the authors were o implement an arm with patients treated with a PCSK9 inhibitor.

Further, is targeting IL-1ß as safe as targeting the LDL-R pathway in terms of side effects? Data from a pooled analysis of the three doses of canakinumab used in the CANTOS trial showed a significantly increase in the deaths from infection compared to those who received placebo [78]. The investigators noted an intriguingly lower risk of cancer mortality with canakinumab than with placebo [79], and so there was a neutral effect on overall mortality.

These data indicate the need for potential novel drugs targeting inflammation in the context of atherosclerosis to further scrutinize the potential negative effects of modulating the immune response which as could result in a reduced ability of the immune system to react toward the infections. Moreover, given that available lipid lowering therapies limit vascular inflammation by reducing LDL levels without relevant effects on infections, clinical approaches should target the axis lipids-lipoproteins-inflammation to achieve a cardiovascular benefit.

\section{Current scenario}

Atherosclerosis is up-to-date recognized as a chronic inflammatory disease where lipids, inflammation and immune response in concert contribute to the pathophysiology of the disease $[80,81]$. IL-1ß has been proposed as a key player linking inflammation to atherogenesis and several other candidates which limit the inflammatory burden of atherosclerosis, as IL-18, CD40/CD40L and CCR2 have been identified [82-88]. In humans, pharmacological targeting of IL-1ß was proven to reduce coronary artery disease [78]; however, other types of interventions, including the inhibition of lipoprotein-associated phospholipase A2 (LP-PLA2) with darapladib [89], or TNF-mediated response with the anti-TNF strategies, have failed in clinical trials to reduce cardiovascular events [90]. These results suggest that inhibiting inflammation "tout court" does not always translate in a beneficial effect on CVD but rather appropriate pathways should be targeted from a clinical perspective. Moreover, a deep understanding of immune-inflammatory mechanisms playing a pathological role versus those which are bystanders of the inflammatory response is needed. Of note, the maintenance of a proper functional immune response is critical to minimize the risk of infection; a better 
identification of patients that will benefit most from II-1beta inhibition should be performed also with the aim of minimizing the increased risk of death associated to sepsis in the CANTOS study.

\section{Future directions}

The increased understanding of molecular basis of cardiovascular diseases is moving clinical practice toward the "precision medicine" shaped on patient with a unique signature of disease. Indeed, although statin decrease cholesterol levels and cardiovascular events, some patients do not fully benefit of the treatment, due to the persistence of a residual risk. Identification of selected biomarkers will help clinicians choosing a personalized therapy based on patient's disease traits; this is the case of hs-CRP that, above 2 $\mathrm{mg} / \mathrm{L}$, provides a solid rationale for the use of an anti-inflammatory strategies on top of lipid-lowering drugs. On the other hand, anti-IL-1ß therapy do not fit all patients and indeed showed to reduce CHD only in those patients which demonstrated a significant reduction of CRP after three months on treatment [91], suggesting a biomarker to track for personalizing the therapy.

\section{Concluding remarks}

The CANTOS trial provides a reliable rationale for targeting inflammation beyond controlling plasma lipids. As cholesterol is known to promote plaque inflammation via inflammasome activation, it is conceivable that, by targeting II-1 beta on the top of lowering plasma lipids, the net effect of cholesterol in promoting atherosclerosis and CVD might be further dampened.

Whether similar conclusions will result from the CIRT trial, aimed at evaluating the cardiovascular benefit of low-dose methotrexate, a drug with systemic anti-inflammatory effects, in patients with prior myocardial infarction and type 2 diabetes or metabolic syndrome remains to be seen [92] but will contribute to understand whether we should "tailor" the anti-inflammatory strategy in the context of atherosclerosis or whether what counts is to limit inflammation. . 
Legends to the Figures

Figure 1. Innate immune memory enhances immune cell activation. Innate immune memory, also referred to as trained immunity, originates from epigenetic reprogramming, i.e. sustained changes in transcription programs following changes in chromatin organization, histone modifications and persistence of microRNAs, which are induced by the first exposure to the dangerous stimulus. In the context of hypercholesterolemia, continuous accumulation of intracellular lipid droplets may shift immune cells to a long term pro-inflammatory phenotype which results in an exceeding immune response after immune challenge.

Figure 2. Meta-inflammation balances functional and metabolic plasticity of immune cells. Obesity, diabetes and atherosclerosis share a typical activation of the immuno-inflammatory response known as meta-inflammation, a chronic low-grade inflammation connecting alterations in systemic metabolism with the impairment in immune cells cellular metabolism and function, i.e. immunometabolism. Metainflammation is associated with a shift of immune cell toward an activated phenotype associated with activation of the inflammasome, aberrant proliferation of lymphocytes and enhanced stimulation of macrophages and dendritic cells. 
1. Tabas I, Lichtman AH: Monocyte-Macrophages and T Cells in Atherosclerosis. Immunity 2017, 47(4):621-634.

2. Lemaire-Ewing S, Lagrost L, Neel D: Lipid rafts: a signalling platform linking lipoprotein metabolism to atherogenesis. Atherosclerosis 2012, 221(2):303-310.

3. Catapano AL, Pirillo A, Bonacina F, Norata GD: HDL in innate and adaptive immunity. Cardiovasc Res 2014, 103(3):372-383.

4. Norata GD, Pirillo A, Ammirati E, Catapano AL: Emerging role of high density lipoproteins as a player in the immune system. Atherosclerosis 2011, 220(1):11-21.

5. Norata GD, Pirillo A, Catapano AL: HDLs, immunity, and atherosclerosis. Curr Opin Lipidol 2011, 22(5):410-416.

6. Varshney $\mathrm{P}$, Yadav $\mathrm{V}$, Saini $\mathrm{N}$ : Lipid rafts in immune signalling: current progress and future perspective. Immunology 2016, 149(1):13-24.

7. Simons K, Gerl MJ: Revitalizing membrane rafts: new tools and insights. Nature reviews Molecular cell biology 2010, 11(10):688-699.

8. Jury EC, Flores-Borja F, Kalsi HS, et al: Abnormal CTLA-4 function in T cells from patients with systemic lupus erythematosus. Eur J Immunol 2010, 40(2):569-578.

9. Eren E, Yates J, Cwynarski K, et al: Location of major histocompatibility complex class II molecules in rafts on dendritic cells enhances the efficiency of T-cell activation and proliferation. Scandinavian journal of immunology 2006, 63(1):7-16.

10. Anderson HA, Hiltbold EM, Roche PA: Concentration of MHC class II molecules in lipid rafts facilitates antigen presentation. Nature immunology 2000, 1(2):156-162.

11. Zhu X, Owen JS, Wilson MD, et al: Macrophage ABCA1 reduces MyD88-dependent Toll-like receptor trafficking to lipid rafts by reduction of lipid raft cholesterol. J Lipid Res 2010, 51(11):3196-3206.

12. Ito A, Hong C, Oka K, et al: Cholesterol Accumulation in CD11C+ Immune Cells Is a Causal and Targetable Factor in Autoimmune Disease. Immunity 2016, 45(6):1311-1326.

13. Foster $\mathrm{GA}, \mathrm{Xu} \mathrm{L}$, Chidambaram $\mathrm{AA}$, et al: CD11c/CD18 Signals Very Late Antigen-4 Activation To Initiate Foamy Monocyte Recruitment during the Onset of Hypercholesterolemia. J Immunol 2015, 195(11):5380-5392.

14. Wu H, Gower RM, Wang $\mathrm{H}$, et al: Functional role of CD11c+ monocytes in atherogenesis associated with hypercholesterolemia. Circulation 2009, 119(20):2708-2717.

15. Xu L, Dai Perrard X, Perrard JL, et al: Foamy monocytes form early and contribute to nascent atherosclerosis in mice with hypercholesterolemia. Arterioscler Thromb Vasc Biol 2015, 35(8):1787-1797.

16. Khan IM, Pokharel Y, Dadu RT, et al: Postprandial Monocyte Activation in Individuals With Metabolic Syndrome. The Journal of clinical endocrinology and metabolism 2016, 101(11):4195-4204.

17. Maxfield FR, Tabas I: Role of cholesterol and lipid organization in disease. Nature 2005, 438(7068):612-621.

18. Plakkal Ayyappan J, Paul A, Goo YH: Lipid droplet-associated proteins in atherosclerosis (Review). Molecular medicine reports 2016, 13(6):4527-4534.

19. Netea MG, Joosten LA, Latz E, et al: Trained immunity: A program of innate immune memory in health and disease. Science 2016, 352(6284):aaf1098.

20. Christ A, Bekkering S, Latz E, Riksen NP: Long-term activation of the innate immune system in atherosclerosis. Semin Immunol 2016, 28(4):384-393. 
21. Pothineni NVK, Subramany S, Kuriakose K, et al: Infections, atherosclerosis, and coronary heart disease. Eur Heart J 2017, 38(43):3195-3201.

22. Bekkering S, Quintin J, Joosten LA, van der Meer JW, Netea MG, Riksen NP: Oxidized lowdensity lipoprotein induces long-term proinflammatory cytokine production and foam cell formation via epigenetic reprogramming of monocytes. Arterioscler Thromb Vasc Biol 2014, 34(8):1731-1738.

23. van der Valk FM, Bekkering S, Kroon J, et al: Oxidized Phospholipids on Lipoprotein(a) Elicit Arterial Wall Inflammation and an Inflammatory Monocyte Response in Humans. Circulation 2016, 134(8):611-624.

24. van der Veen JN, Kruit JK, Havinga R, et al: Reduced cholesterol absorption upon PPARdelta activation coincides with decreased intestinal expression of NPC1L1. J Lipid Res 2005, 46(3):526-534.

25. Janoudi A, Shamoun FE, Kalavakunta JK, Abela GS: Cholesterol crystal induced arterial inflammation and destabilization of atherosclerotic plaque. Eur Heart J 2016, 37(25):19591967.

26. Samstad EO, Niyonzima N, Nymo S, et al: Cholesterol crystals induce complementdependent inflammasome activation and cytokine release. J Immunol 2014, 192(6):28372845.

27. Abela GS, Kalavakunta JK, Janoudi A, et al: Frequency of Cholesterol Crystals in Culprit Coronary Artery Aspirate During Acute Myocardial Infarction and Their Relation to Inflammation and Myocardial Injury. The American journal of cardiology 2017, 120(10):1699-1707.

28. Mason RP, Jacob RF: Membrane microdomains and vascular biology: emerging role in atherogenesis. Circulation 2003, 107(17):2270-2273.

29. Martinon F, Burns K, Tschopp J: The inflammasome: a molecular platform triggering activation of inflammatory caspases and processing of prolL-beta. Molecular cell 2002, 10(2):417-426.

30. Karasawa T, Takahashi M: Role of NLRP3 Inflammasomes in Atherosclerosis. J Atheroscler Thromb 2017, 24(5):443-451.

31. Duewell $\mathrm{P}$, Kono $\mathrm{H}$, Rayner KJ, et al: NLRP3 inflammasomes are required for atherogenesis and activated by cholesterol crystals. Nature 2010, 464(7293):1357-1361.

32. Menu P, Pellegrin M, Aubert JF, et al: Atherosclerosis in ApoE-deficient mice progresses independently of the NLRP3 inflammasome. Cell death \& disease 2011, 2:e137.

33. Sheedy FJ, Grebe A, Rayner KJ, et al: CD36 coordinates NLRP3 inflammasome activation by facilitating intracellular nucleation of soluble ligands into particulate ligands in sterile inflammation. Nature immunology 2013, 14(8):812-820.

34. Hendrikx T, Jeurissen ML, van Gorp PJ, et al: Bone marrow-specific caspase-1/11 deficiency inhibits atherosclerosis development in $\operatorname{Ld} \operatorname{lr}(-/-)$ mice. The FEBS journal 2015, 282(12):2327-2338.

35. Freigang S, Ampenberger F, Weiss A, et al: Fatty acid-induced mitochondrial uncoupling elicits inflammasome-independent IL-1alpha and sterile vascular inflammation in atherosclerosis. Nature immunology 2013, 14(10):1045-1053.

36. Tan HW, Liu X, Bi XP, et al: IL-18 overexpression promotes vascular inflammation and remodeling in a rat model of metabolic syndrome. Atherosclerosis 2010, 208(2):350-357.

37. de Nooijer R, von der Thusen JH, Verkleij CJ, et al: Overexpression of IL-18 decreases intimal collagen content and promotes a vulnerable plaque phenotype in apolipoprotein-Edeficient mice. Arterioscler Thromb Vasc Biol 2004, 24(12):2313-2319. 
38. Kirii H, Niwa $T$, Yamada $\mathrm{Y}$, et al: Lack of interleukin-1beta decreases the severity of atherosclerosis in ApoE-deficient mice. Arterioscler Thromb Vasc Biol 2003, 23(4):656-660.

39. Usui $F$, Shirasuna $\mathrm{K}$, Kimura $\mathrm{H}$, et al: Critical role of caspase-1 in vascular inflammation and development of atherosclerosis in Western diet-fed apolipoprotein E-deficient mice. Biochem Biophys Res Commun 2012, 425(2):162-168.

40. Hotamisligil GS: Inflammation, metaflammation and immunometabolic disorders. Nature 2017, 542(7640):177-185.

41. Norata GD, Caligiuri G, Chavakis $T$, et al: The cellular and molecular basis of translational immunometabolism. Immunity 2015, 43(3):421-434.

42. Zhou R, Tardivel A, Thorens B, Choi I, Tschopp J: Thioredoxin-interacting protein links oxidative stress to inflammasome activation. Nature immunology 2010, 11(2):136-140.

43. Masters SL, Latz E, O'Neill LA: The inflammasome in atherosclerosis and type 2 diabetes. Science translational medicine 2011, 3(81):81ps17.

44. Wen $H$, Gris D, Lei $Y$, et al: Fatty acid-induced NLRP3-ASC inflammasome activation interferes with insulin signaling. Nature immunology 2011, 12(5):408-415.

45. Murphy AJ, Akhtari M, Tolani S, et al: ApoE regulates hematopoietic stem cell proliferation, monocytosis, and monocyte accumulation in atherosclerotic lesions in mice. J Clin Invest 2011, 121(10):4138-4149.

46. Westerterp M, Gourion-Arsiquaud S, Murphy AJ, et al: Regulation of hematopoietic stem and progenitor cell mobilization by cholesterol efflux pathways. Cell stem cell 2012, 11(2):195-206.

47. Yvan-Charvet L, Pagler T, Gautier EL, et al: ATP-binding cassette transporters and HDL suppress hematopoietic stem cell proliferation. Science 2010, 328(5986):1689-1693.

48. Wang Y, Viscarra J, Kim SJ, Sul HS: Transcriptional regulation of hepatic lipogenesis. Nature reviews Molecular cell biology 2015, 16(11):678-689.

49. Im SS, Yousef L, Blaschitz C, et al: Linking lipid metabolism to the innate immune response in macrophages through sterol regulatory element binding protein-1a. Cell Metab 2011, 13(5):540-549.

50. **Oishi Y, Spann NJ, Link VM, et al: SREBP1 Contributes to Resolution of Pro-inflammatory TLR4 Signaling by Reprogramming Fatty Acid Metabolism. Cell Metab 2017, 25(2):412-427.

**This paper highlights the role of SREBP1c in macrophages during the resolution phase thus providing novel insights into lipid requirements according to different functional stage of immune cells

51. Ito A, Hong C, Rong $X$, et al: LXRs link metabolism to inflammation through Abca1dependent regulation of membrane composition and TLR signaling. eLife 2015, 4:e08009.

52. Brown MS, Goldstein JL: Lipoprotein receptors and genetic control of cholesterol metabolism in cultured human cells. Die Naturwissenschaften 1975, 62(8):385-389.

53. Bensinger SJ, Bradley MN, Joseph SB, et al: LXR signaling couples sterol metabolism to proliferation in the acquired immune response. Cell 2008, 134(1):97-111.

54. Kidani $\mathrm{Y}$, Elsaesser $\mathrm{H}$, Hock $\mathrm{MB}$, et al: Sterol regulatory element-binding proteins are essential for the metabolic programming of effector T cells and adaptive immunity. Nature immunology 2013, 14(5):489-499.

55. Buck MD, Sowell RT, Kaech SM, Pearce EL: Metabolic Instruction of Immunity. Cell 2017, 169(4):570-586.

56. Haas R, Smith J, Rocher-Ros V, et al: Lactate Regulates Metabolic and Pro-inflammatory Circuits in Control of T Cell Migration and Effector Functions. PLoS biology 2015, 13(7):e1002202. 
57. *Mauro C, Smith J, Cucchi D, et al: Obesity-Induced Metabolic Stress Leads to Biased Effector Memory CD4+ T Cell Differentiation via PI3K p110delta-Akt-Mediated Signals. Cell Metab 2017, 25(3):593-609.

*This paper provides evidence of a direct role of saturated free fatty acids in priming CD4 T cell differentiation and trafficking to inflammatory sites independently of the metabolic status of the host

58. Ni K, O'Neill HC: The role of dendritic cells in T cell activation. Immunology and cell biology 1997, 75(3):223-230.

59. Steinman RM: Linking innate to adaptive immunity through dendritic cells. Novartis Foundation symposium 2006, 279:101-109; discussion 109-113, 216-109.

60. Zernecke A: Dendritic cells in atherosclerosis: evidence in mice and humans. Arterioscler Thromb Vasc Biol 2015, 35(4):763-770.

61. **Westerterp M, Gautier EL, Ganda A, et al: Cholesterol Accumulation in Dendritic Cells Links the Inflammasome to Acquired Immunity. Cell Metab 2017, 25(6):1294-1304 e1296.

$* *$ This paper identifies an essential role of cholesterol efflux pathway mediated by $A B C A 1 / G 1$ in maintaining immune tolerance

62. Charles-Schoeman C: Cardiovascular disease and rheumatoid arthritis: an update. Curr Rheumatol Rep 2012, 14(5):455-462.

63. Ronda N, Favari E, Borghi MO, et al: Impaired serum cholesterol efflux capacity in rheumatoid arthritis and systemic lupus erythematosus. Ann Rheum Dis 2014, 73(3):609615.

64. Wang SH, Yuan SG, Peng DQ, Zhao SP: HDL and ApoA-I inhibit antigen presentationmediated $T$ cell activation by disrupting lipid rafts in antigen presenting cells. Atherosclerosis 2012, 225(1):105-114.

65. Baigent C, Blackwell L, Emberson J, et al: Efficacy and safety of more intensive lowering of LDL cholesterol: a meta-analysis of data from 170,000 participants in 26 randomised trials. Lancet 2010, 376(9753):1670-1681.

66. Baigent $C$, Keech A, Kearney PM, et al: Efficacy and safety of cholesterol-lowering treatment: prospective meta-analysis of data from 90,056 participants in 14 randomised trials of statins. Lancet 2005, 366(9493):1267-1278.

67. Oesterle A, Laufs U, Liao JK: Pleiotropic Effects of Statins on the Cardiovascular System. Circ Res 2017, 120(1):229-243.

68. Boekholdt SM, Hovingh GK, Mora S, et al: Very low levels of atherogenic lipoproteins and the risk for cardiovascular events: a meta-analysis of statin trials. Journal of the American College of Cardiology 2014, 64(5):485-494.

69. Kinlay S: Low-density lipoprotein-dependent and -independent effects of cholesterollowering therapies on C-reactive protein: a meta-analysis. Journal of the American College of Cardiology 2007, 49(20):2003-2009.

70. Catapano AL, Pirillo A, Norata GD: Vascular inflammation and low-density lipoproteins: is cholesterol the link? A lesson from the clinical trials. Br J Pharmacol 2017, 174(22):39733985.

71. Noveck R, Stroes ES, Flaim JD, et al: Effects of an antisense oligonucleotide inhibitor of Creactive protein synthesis on the endotoxin challenge response in healthy human male volunteers. J Am Heart Assoc 2014, 3(4).

72. Lane $\mathrm{T}$, Wassef $\mathrm{N}$, Poole $\mathrm{S}$, et al: Infusion of pharmaceutical-grade natural human Creactive protein is not proinflammatory in healthy adult human volunteers. Circ Res 2014, 114(4):672-676. 
73. Elliott $P$, Chambers JC, Zhang W, et al: Genetic Loci associated with C-reactive protein levels and risk of coronary heart disease. Jama 2009, 302(1):37-48.

74. Zacho J, Tybjaerg-Hansen A, Jensen JS, Grande P, Sillesen H, Nordestgaard BG: Genetically elevated C-reactive protein and ischemic vascular disease. N Engl J Med 2008, 359(18):1897-1908.

75. Wensley F, Gao P, Burgess $S$, et al: Association between $C$ reactive protein and coronary heart disease: mendelian randomisation analysis based on individual participant data. Bmj 2011, 342:d548.

76. Sabatine MS, Giugliano RP, Keech AC, et al: Evolocumab and Clinical Outcomes in Patients with Cardiovascular Disease. N Engl J Med 2017, 376(18):1713-1722.

77. Nicholls SJ, Puri R, Anderson T, et al: Effect of Evolocumab on Progression of Coronary Disease in Statin-Treated Patients: The GLAGOV Randomized Clinical Trial. Jama 2016, 316(22):2373-2384.

78. **Ridker PM, Everett BM, Thuren T, et al: Antiinflammatory Therapy with Canakinumab for Atherosclerotic Disease. N Engl J Med 2017, 377(12):1119-1131.

**This trial shows the cardiovascular benefit of inhibiting IL-1ß, thus supporting the inflammatory hypothesis of atherosclerosis

79. Ridker PM, MacFadyen JG, Thuren T, et al: Effect of interleukin-1beta inhibition with canakinumab on incident lung cancer in patients with atherosclerosis: exploratory results from a randomised, double-blind, placebo-controlled trial. Lancet 2017, 390(10105):18331842.

80. Tall AR, Yvan-Charvet L: Cholesterol, inflammation and innate immunity. Nature reviews Immunology 2015, 15(2):104-116.

81. Rosenfeld ME: Inflammation and atherosclerosis: direct versus indirect mechanisms. Current opinion in pharmacology 2013, 13(2):154-160.

82. Elhage $\mathrm{R}$, Jawien $\mathrm{J}$, Rudling $\mathrm{M}$, et al: Reduced atherosclerosis in interleukin-18 deficient apolipoprotein E-knockout mice. Cardiovasc Res 2003, 59(1):234-240.

83. Li JM, Eslami MH, Rohrer MJ, et al: Interleukin 18 binding protein (IL18-BP) inhibits neointimal hyperplasia after balloon injury in an atherosclerotic rabbit model. Journal of vascular surgery 2008, 47(5):1048-1057.

84. Hueso M, De Ramon L, Navarro E, et al: Silencing of CD40 in vivo reduces progression of experimental atherogenesis through an NF-kappaB/miR-125b axis and reveals new potential mediators in the pathogenesis of atherosclerosis. Atherosclerosis 2016, 255:8089.

85. Mach F, Schonbeck U, Sukhova GK, Atkinson E, Libby P: Reduction of atherosclerosis in mice by inhibition of CD40 signalling. Nature 1998, 394(6689):200-203.

86. Schonbeck U, Sukhova GK, Shimizu K, Mach F, Libby P: Inhibition of CD40 signaling limits evolution of established atherosclerosis in mice. Proc Natl Acad Sci U S A 2000, 97(13):7458-7463.

87. Bot I, Ortiz Zacarias NV, de Witte WE, et al: A novel CCR2 antagonist inhibits atherogenesis in apoE deficient mice by achieving high receptor occupancy. Scientific reports 2017, 7(1):52.

88. Olzinski AR, Turner GH, Bernard RE, et al: Pharmacological inhibition of C-C chemokine receptor 2 decreases macrophage infiltration in the aortic root of the human $\mathrm{C}-\mathrm{C}$ chemokine receptor 2/apolipoprotein E-/- mouse: magnetic resonance imaging assessment. Arterioscler Thromb Vasc Biol 2010, 30(2):253-259.

89. White HD, Held C, Stewart R, et al: Darapladib for preventing ischemic events in stable coronary heart disease. N Engl J Med 2014, 370(18):1702-1711. 
90. Silva LC, Ortigosa LC, Benard G: Anti-TNF-alpha agents in the treatment of immunemediated inflammatory diseases: mechanisms of action and pitfalls. Immunotherapy 2010, 2(6):817-833.

91. Ridker PM, MacFadyen JG, Everett BM, et al: Relationship of C-reactive protein reduction to cardiovascular event reduction following treatment with canakinumab: a secondary analysis from the CANTOS randomised controlled trial. Lancet 2017.

92. Everett $\mathrm{BM}$, Pradhan $\mathrm{AD}$, Solomon $\mathrm{DH}$, et al: Rationale and design of the Cardiovascular Inflammation Reduction Trial: a test of the inflammatory hypothesis of atherothrombosis. Am Heart J 2013, 166(2):199-207 e115. 
Figure 1

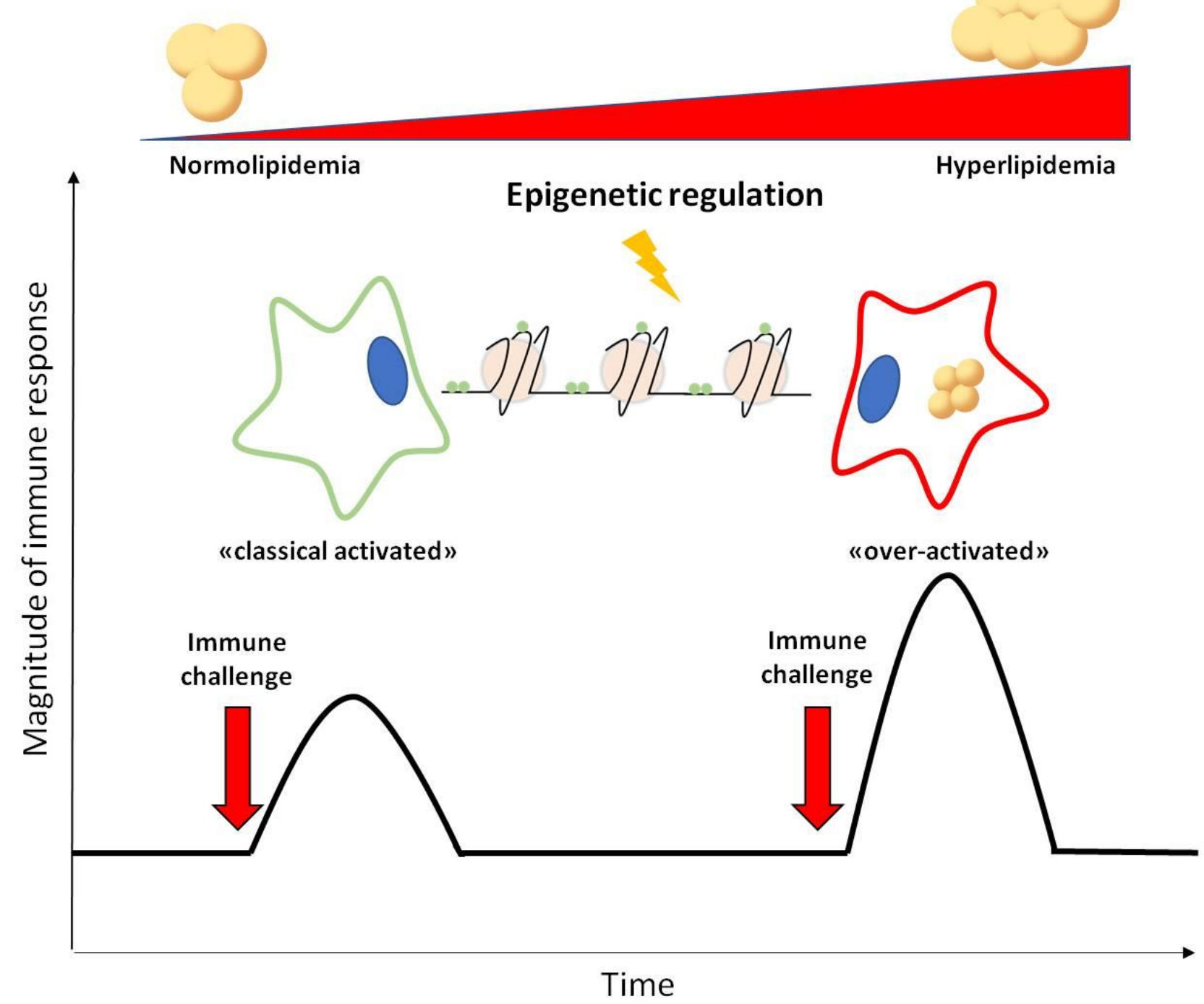


Figure 2

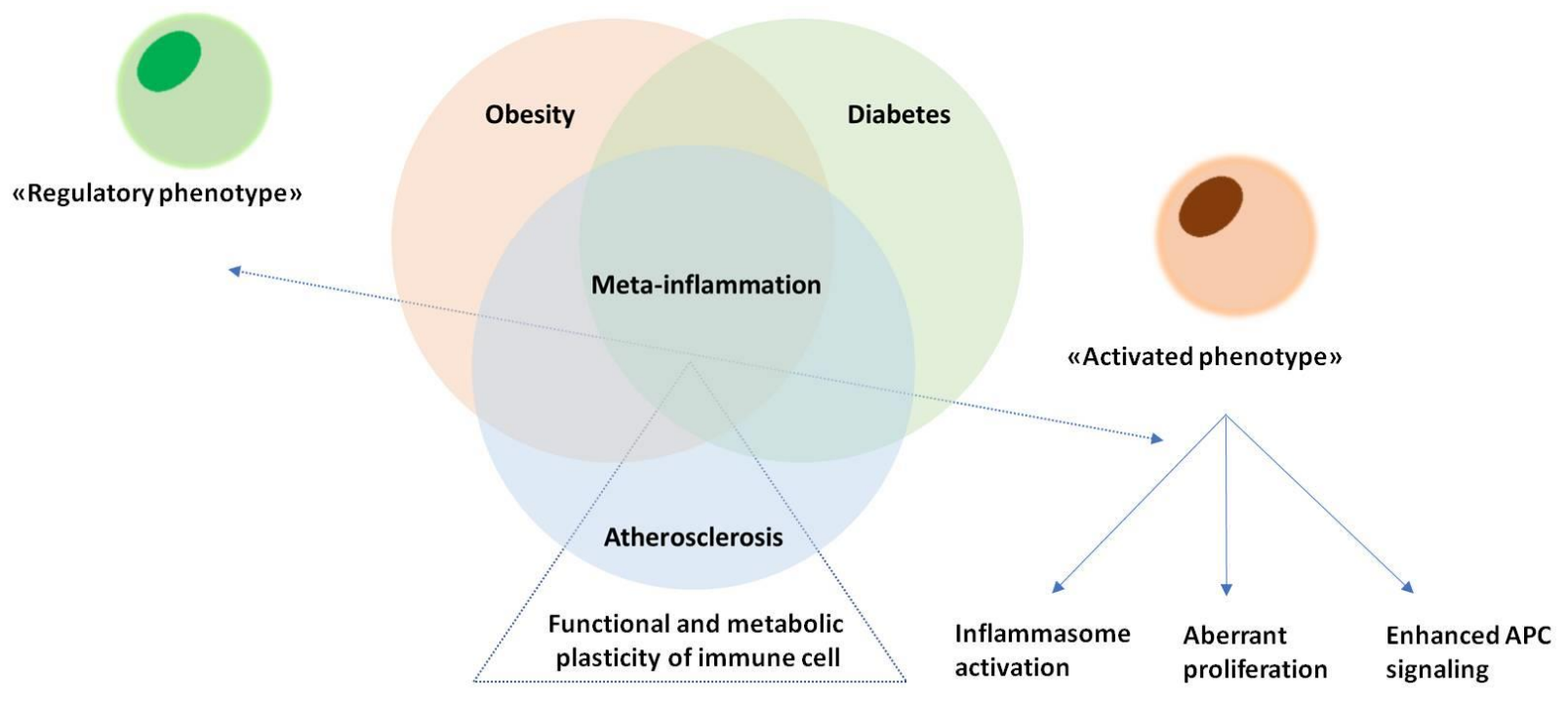

